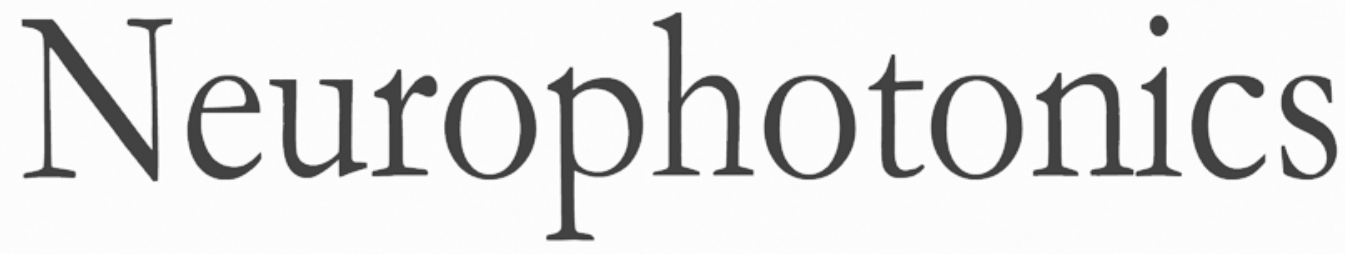

\title{
Prefrontal hemodynamic changes measured using near-infrared spectroscopy during the Valsalva maneuver in patients with orthostatic intolerance
}

\author{
Yoo Hwan Kim \\ Zephaniah Phillips V \\ Seung-ho Paik \\ Nam-Joon Jeon \\ Beop-Min Kim \\ Byung-Jo Kim
}




\title{
Prefrontal hemodynamic changes measured using near-infrared spectroscopy during the Valsalva maneuver in patients with orthostatic intolerance
}

\author{
Yoo Hwan Kim, ${ }^{a, b, \dagger}$ Zephaniah Phillips V, ${ }^{c, \dagger}$ Seung-ho Paik, ${ }^{c}$ Nam-Joon Jeon, ${ }^{d}$ Beop-Min Kim, ${ }^{c, *, \ddagger}$ and \\ Byung-Jo Kim ${ }^{b, e, *, \neq}$ \\ aHallym University Medical Center, Department of Neurology, Seoul, Republic of Korea \\ ${ }^{b} K o r e a$ University Medical Center, Department of Neurology, Seoul, Republic of Korea \\ 'Korea University College of Health Science, Department of Bioconvergence Engineering, Seoul, Republic of Korea \\ ${ }^{d}$ Korea University Anam Hospital, Neurophysiology Laboratory, Seoul, Republic of Korea

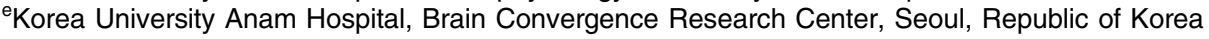

\begin{abstract}
The Valsalva maneuver (VM) with beat-to-beat blood pressure and heart rate monitoring are used to evaluate orthostatic intolerance $(\mathrm{OI})$. However, they lack the ability to detect cerebral hemodynamic changes, which may be a cause of Ol symptoms. Therefore, we utilized near-infrared spectroscopy during VM. Patients with Ol symptoms and normal healthy subjects were recruited. Patients were subgrouped according to VM results: patients with normal VM (NVM) and abnormal VM (AbVM). Oxyhemoglobin ( $\mathrm{HbO})$, deoxyhemoglobin, and total hemoglobin changes were measured at four different source-detector distances (SD) $(15,30,36$, and $45 \mathrm{~mm}$ ), and latency, amplitude, duration, and integrated total signal were calculated. Those parameters were compared between a normal healthy control $(\mathrm{HC})$ group and the two Ol patient subgroups. We found that $\mathrm{HbO}$ increment latency at 30-mm SD in the HC, NVM, and AbVM groups was as follows: $0.39 \pm 0.23 \mathrm{~s}, 2.79 \pm 0.36 \mathrm{~s}$, and $8.14 \pm 0.55 \mathrm{~s}$, respectively $(p<0.05)$. Among the four parameters we evaluated, latency of $\mathrm{HbO}$ increment was the best marker for differentiating OI. ๑ 2018 Society of Photo-Optical Instrumentation Engineers (SPIE) [DOI: 10.1117/1.NPh.5.1 .015002]
\end{abstract}

Key words: orthostatic intolerance; spectroscopy; near-infrared; Valsalva maneuver.

Paper 17108RR received Jul. 20, 2017; accepted for publication Jan. 4, 2018; published online Jan. 26, 2018.

\section{Introduction}

Various autonomic function tests (AFTs) have been used to evaluate autonomic dysfunction; however, only a few tests have been validated as clinically quantitative methods to evaluate major autonomic domains, including sudomotor, cardiovagal, and adrenergic. ${ }^{1}$ The Valsalva maneuver (VM) is one of the validated quantifying AFTs. The VM is easy to perform and is a useful method for simultaneously evaluating both sympathetic adrenergic functions using blood pressure (BP) responses and parasympathetic cardiovagal functions using heart rate (HR) responses. $^{2}$ Moreover, Valsalva activity evokes various symptoms related to dysregulation of the autonomic nervous system (ANS), such as dizziness, light headedness, blurred vision, and palpitation. The maneuver can be performed with prolonged forced exhalation through a closed airway and can occur naturally during routine activities, such as heavy lifting, straining on the toilet, or vomiting. Overall hemodynamic autoregulation during VM is a key factor in preventing the development of significant clinical symptoms such as orthostatic intolerance (OI), which is one of the representative manifestations of autonomic dysfunction or syncope. ${ }^{3,4}$

However, BP and HR monitoring alone is not enough to detect pathologic dysfunction of the ANS. In addition, the

\footnotetext{
${ }^{\star}$ Address all correspondence to: Beop-Min Kim, E-mail: bmk515@korea.ac.kr; Byung-Jo Kim, E-mail: nukbj@korea.ac.kr

†These authors contributed equally to this study.

¥These corresponding authors contributed equally to this study.
}

mechanisms behind VM that determine the symptoms caused by autonomic dysfunction are still unclear. Many previous studies have suggested a decrease in cerebral perfusion evoked by autonomic reflexes during Valsalva activity as a possible mechanism. $^{5-9}$

To fully explore the hemodynamic changes during VM, we chose to use near-infrared spectroscopy (NIRS) to monitor the relative concentration changes in oxyhemoglobin $(\mathrm{HbO})$, deoxyhemoglobin $(\mathrm{Hb})$, and total hemoglobin (HbT). NIRS has been shown to be a useful tool for evaluating cerebral hemodynamics during various dynamic activities. In particular, NIRS has successfully been used to observe cerebral hemodynamics regulated by AFTs. ${ }^{10-13}$ Various studies have been conducted to explore cerebral hemodynamics during VM for healthy subjects as monitored by NIRS. Perry et al. ${ }^{9}$ reported that greater VM intensity affects cerebral hemodynamics with reduced oxygenation during each VM phase. The main finding of Tsubaki et al. ${ }^{14}$ was that $\mathrm{HbO}$ measured by NIRS was strongly correlated with mean arterial pressure (MAP) during VM. Various other relationships between $\mathrm{VM}$ and cerebral autoregulation were reported in healthy subjects using NIRS. ${ }^{15-17}$ However, the effects of autonomic dysfunction on cerebral hemodynamics during VM have yet to be thoroughly investigated. In normal healthy people, there is a close relationship between cerebral hemodynamic autoregulation and VM, but we hypothesized that, since autonomic dysfunction can have a profound effect on the delivery and regulation of cerebral hemodynamics, ${ }^{18}$ the VM

2329-423X/2018/\$25.00 (C 2018 SPIE 
hemodynamics of patients with autonomic dysfunction might differ from those of healthy subjects.

In this study, we explore the effectiveness of NIRS for proper assessment of patients with OI through various metrics that quantify cerebral hemodynamic activity during VM. In this way, we hoped to present NIRS as a possible method for understanding the mechanisms that govern significant clinical symptoms involving dysregulation of the ANS.

\section{Materials and Methods}

\subsection{Ethics Statement}

All participants gave written informed consent before study inclusion. All procedures were performed in accordance with the Declaration of Helsinki and were approved by the Korea University Medical Center Institutional Review Board (IRB No. ED15363).

\subsection{Subjects}

Patients with symptoms of OI were recruited from January 2016 to June 2016. For study accuracy, we rigorously adjusted the inclusion criteria. Inclusion criteria were (1) symptoms suggesting OI (memory loss, visual difficulties, light headedness, headache, fatigue, increases or decreases in BP, weakness, nausea and abdominal pain, sweating, tremulousness, and exercise intolerance), ${ }^{19}$ (2) no history of central nervous system diseases, including stroke, Parkinson's disease, and Alzheimer's dementia, and (3) no history of significant head injury, alcohol, or psychotropic drug abuse. Demographic and clinical data, including age, gender, and comorbid chronic diseases, were obtained. Normal healthy control (HC) volunteers who did not have any medical history that might affect ANS were recruited. Healthy subjects who had any abnormal findings in the AFTs of this study protocol and had any OI symptoms according to the OI questionnaires were excluded from final analysis. In addition, subjects with arrhythmia were excluded because of the possibility of VM measurement error. A total of 31 patients with OI and 9 healthy subjects who completed all study protocols were recruited. However, four patients and three healthy subjects who showed arrhythmia, which made parameters unreliable, were excluded from final analysis. Two patients who had NIRS signals that were not sufficient to be analyzed due to excessive motion artifacts were excluded. Another three healthy subjects were excluded due to abnormal VM (AbVM) parameter findings: two subjects showed abnormal Valsalva ratios (VRs) and one subject showed abnormal cardiovagal barosensitivity (BRSv). Finally, data from 25 patients and 3 normal HCs were analyzed.

\subsection{Study Design}

All participants taking medications affecting autonomic function were asked to discontinue their medicine for at least $24 \mathrm{~h}$ prior to VM. To minimize the effects of confounds, VM was controlled and regulated by the standard electrodiagnostic laboratory environment. ${ }^{20}$ Tests were performed in the following sequence: (1) autonomic dysfunction self-questionnaires were completed by the patient and (2) VMs were conducted while attaching the NIRS probe on the forehead to investigate cerebral hemodynamics.

\subsection{Autonomic Dysfunction Questionnaires}

Self-report questionnaires were administered. Autonomic dysfunction was measured using the Korean version of the Composite Autonomic Symptom Score 31 (K-COMPASS 31) consisting of 31 items that assess the following categories: 4 items for OI, 3 items for vasomotor, 4 items for secretomotor, 12 items for gastrointestinal, 3 items for bladder, and 5 items for pupillomotor. The sum of items from each category and the sum of all 31 items were used for analysis. Of note, higher scores indicate more severe autonomic dysfunction. ${ }^{21} \mathrm{We}$ also only scored the four items related to OI separately among the many items of the K-COMPASS 31 questionnaire.

\subsection{Valsalva Maneuver}

The VM was performed with Finometer equipment (Finapres Medical Systems BV, Amsterdam, The Netherlands) with a cuff placed on the middle finger. Subjects blew through a mouthpiece attached to a manometer and maintained a pressure of $40 \mathrm{~mm} \mathrm{Hg}$ for $15 \mathrm{~s}$ at rest and in the recumbent position. VM is a difficult procedure to perform consecutively, so each trial was followed by a rest period of $2 \mathrm{~min}$, with testing repeated until two responses with similar beat-to-beat BP and HR recordings were obtained. The VM trial that showed the correct four phases of VM in the BP recordings was chosen for further NIRS analysis. Therefore, there was only one VM trial per subject in which BP and NIRS data were used for analysis. The following BP metrics were determined for each patient. (1) VR was calculated as the maximum HR during the maneuver in phase II divided by the lowest HR in phase IV. The VR of each subject was compared with age-specific reference values. ${ }^{2,22}$ (2) Pressure recovery time (PRT) was defined as the time from the lowest systolic BP (SBP) during the valley of phase III (start of phase IV) to the return of SBP to baseline before overshooting of SBP during phase IV. ${ }^{1,23}$ (3) Alternative adrenergic barosensitivity $\left(\mathrm{BRSa}_{1}\right)$ was defined as the sum of the SBP decrement during early phase II and two-thirds of the SBP decrement in phase III divided by PRT. ${ }^{24,25}$ (4) BRSv was determined as the regression slope of the $R-R$ interval (milliseconds) over SBP during early phase II. ${ }^{24-27}$ Using the cutoff value for each parameter, patients with an abnormal result in any of the four parameters were placed into the AbVM group.

\subsection{Near-Infrared Spectroscopy}

A custom-built 108-channel NIRS system was administered to the subject pool to monitor cerebral hemodynamics during VM (Fig. 1). The NIRS probe was built to span the subject's entire forehead and was secured in place by a medical band. The center bottom of the NIRS probe was aligned with the FpZ point, and the left and right corners were aligned with the F7 and F8 points, according to the International 10-10 system. This alignment has been shown to be appropriate for monitoring cerebral oxygenation changes in the prefrontal area. ${ }^{28,29}$ The experiment administrator visually ensured good contact for all sources and detectors before the experiment started. In addition, the NIRS software included a module that identified saturated or low-signal channels based on an empirically tested range of light intensities. This was confirmed by the administrator before the tests began. The subjects were instructed to avoid large head motions that might induce motion artifacts in the NIRS signal. The NIRS 


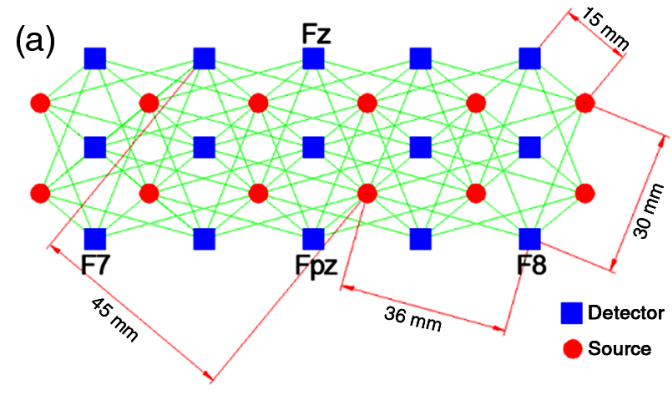

(b)

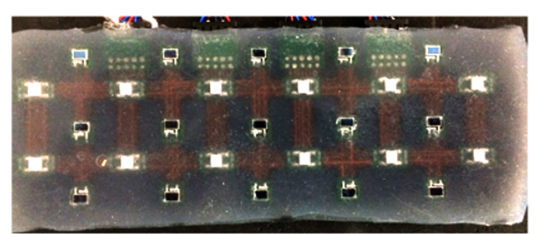

Fig. 1 A 108-channel matrix of NIRS. (a) Shows the four different source-detector separations $(15,30,36$, and $45 \mathrm{~mm}$ ) with the bottom of the probe aligned with the F7-Fpz-F8 line on the subject's head. (b) The actual NIRS probe consisted of 15 photodetectors and 12 LED sources.

probe remained on the subject as they performed multiple VM trials.

The NIRS probe was attached to the forehead area because of the lack of hair that could significantly degrade signal quality. The probe consisted of 15 photodetectors and 12 light-emitting diodes (LED) sources. Each of the LED sources alternated between wavelengths of 760 and $830 \mathrm{~nm}$ at a temporal frequency of $5 \mathrm{~Hz}$ for scanning the entire forehead. The power emitted from the LED sources was $\sim 4 \mathrm{~mW}$. The 108 channels came from the 108-unique source-detector pairings with different source-detector distances (SD) of $15 \mathrm{~mm}$ (40 channels), $30 \mathrm{~mm}$ (20 channels), $36 \mathrm{~mm}$ (32 channels), and $45 \mathrm{~mm}$ (16 channels). The channel matrix is shown in Fig. 1; the green line connecting a source and detector signifies one channel. The separation between source and detector determines the penetration depth, with the greatest sensitivity to gray matter changes being shown at a separation of $30 \mathrm{~mm}$ or greater. ${ }^{30}$ Before the calculation of the NIRS metrics, the data were lowpassed filtered (cutoff frequency of $0.2 \mathrm{~Hz}$ ) and smoothed with a 5-point moving average filter, and the first point of the calculated $\mathrm{HbO}, \mathrm{Hb}$, and $\mathrm{HbT}$ signals was realigned to zero. In this study, we analyzed changes in $\mathrm{HbO}, \mathrm{Hb}$, and $\mathrm{HbT}$ to determine whether the observed hemodynamic response varied according to SD.

Resolving for changes in optical density or the log ratio of baseline intensity, $I_{o}$ and transient intensity $I$ changes at the two wavelengths (760 and $830 \mathrm{~nm}$ ) allowed accurate measurement of relative changes in $\mathrm{HbO}, \mathrm{Hb}$, and $\mathrm{HbT}^{31}$ with $\mathrm{HbT}$ being proportional to blood volume. ${ }^{32}$ In short, the relative concentration change in $\mathrm{HbO}$ and $\mathrm{Hb}$ can be resolved using the modified Beer-Lambert's law ${ }^{33}$

$$
\begin{gathered}
\Delta \mathrm{HbO}=\frac{\varepsilon_{\mathrm{Hb}}^{\lambda_{2}} \log \left(\frac{I_{o}}{I}\right)^{\lambda_{1}}-\varepsilon_{\mathrm{Hb}}^{\lambda_{1}} \log \left(\frac{I_{o}}{I}\right)^{\lambda_{2}}}{L\left(\varepsilon_{\mathrm{Hb}}^{\lambda_{2}} \varepsilon_{\mathrm{HbO}}^{\lambda_{1}}-\varepsilon_{\mathrm{Hb}}^{\lambda_{1}} \varepsilon_{\mathrm{HbO}}^{\lambda_{2}}\right)}, \\
\Delta \mathrm{Hb}=\frac{\varepsilon_{\mathrm{HbO}}^{\lambda_{1}} \log \left(\frac{I_{o}}{I}\right)^{\lambda_{2}}-\varepsilon_{\mathrm{HbO}}^{\lambda_{2}} \log \left(\frac{I_{o}}{I}\right)^{\lambda_{1}}}{L\left(\varepsilon_{\mathrm{Hb}}^{\lambda_{2}} \varepsilon_{\mathrm{HbO}}^{\lambda_{1}}-\varepsilon_{\mathrm{Hb}}^{\lambda_{1}} \varepsilon_{\mathrm{HbO}}^{\lambda_{2}}\right)},
\end{gathered}
$$

where $\Delta \mathrm{HbO}$ and $\Delta \mathrm{Hb}$ are the change in $\mathrm{HbO}$ and $\mathrm{Hb}$, respectively, from the baseline state. The baseline intensity was set to the average intensity of the first 100 acquired points before the VM started. $L$ is the optical pathlength, and $\varepsilon_{\mathrm{HbO}, \mathrm{Hb}}^{\lambda_{1}}$ and $\varepsilon_{\mathrm{HbO}, \mathrm{Hb}}^{\lambda_{2}}$ are the extinction coefficients for $\mathrm{HbO}$ and $\mathrm{Hb}$ for the two wavelengths $\lambda_{1}=760 \mathrm{~nm}$ and $\lambda_{2}=830 \mathrm{~nm}$, respectively, taken from Ref. 34. A full review of NIRS methodology and the terms above can be found elsewhere. ${ }^{35}$

\subsection{NIRS Parameters}

Upon preliminary review of the hemodynamic changes during VM, NIRS parameters were determined to compare the hemodynamic changes between subjects. These parameters were latency, duration, and amplitude calculated for $\mathrm{HbO}$ and $\mathrm{Hb}$, and the integrated signal calculated for HbT. Latency was defined as the time between reaching the appropriate pressure to perform the VM ( 40-mm Hg exhalation pressure) and the beginning of the NIRS hemodynamic response. The amplitude was defined as the maximum concentration change in the calculated NIRS response. Duration was defined as the width of the NIRS
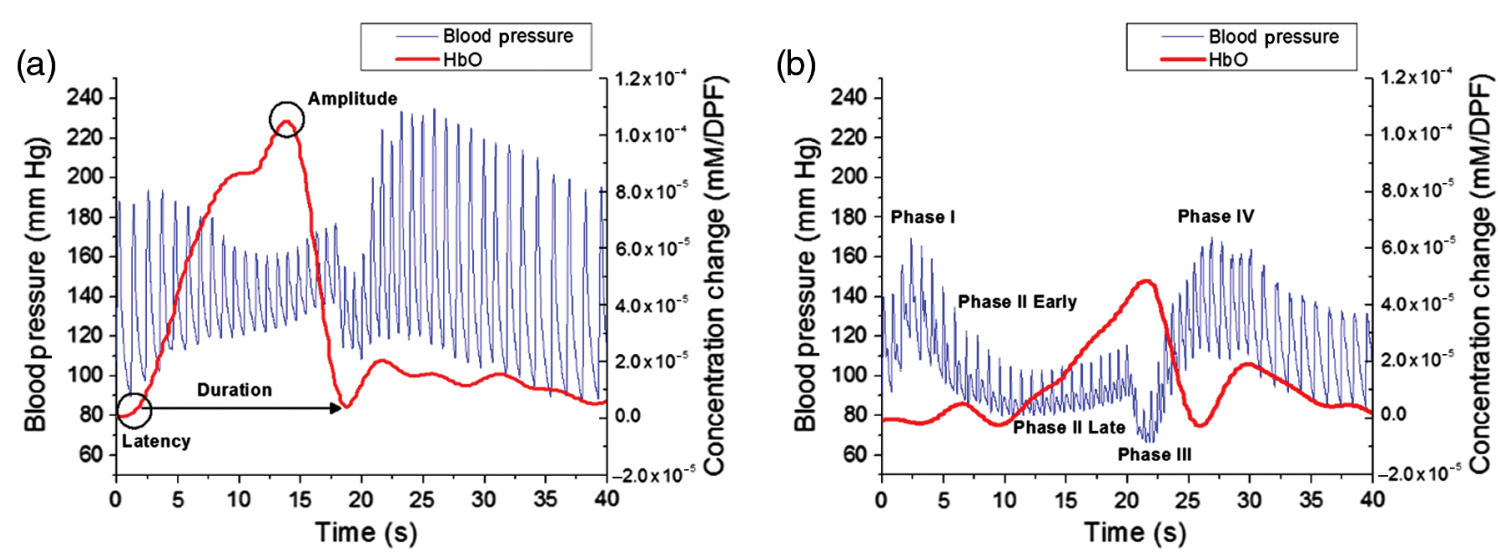

Fig. 2 NIRS response during VM. Comparison of BP (blue) and $\mathrm{HbO}$ (red) changes for (a) an $\mathrm{HC}$ and (b) an AbVM patient with OI. In addition, a visual depiction of calculated metrics is shown. (a) The NIRS parameters (latency, amplitude, and duration) and (b) four phases of the VM (according to BP measurements) are shown. 
Table 1 Subject demographic and clinical characteristics.

$\operatorname{NVM}(n=9) \quad \operatorname{AbVM}(n=16) \quad$ Normal healthy $(n=3) \quad p$-value

Clinical features

\begin{tabular}{|c|c|c|c|c|}
\hline Male (\%) & 22.2 & 50 & 66.7 & 0.27 \\
\hline Age (year) & $66.00(31,80)$ & $49.00(22,79)$ & $66.00(66,74)$ & 0.11 \\
\hline Height (m) & $1.61(1.54,1.79)$ & $1.62(1.49,1.75)$ & $1.61(1.56,1.65)$ & 0.86 \\
\hline Weight (kg) & $61.00(53,94)$ & $60.00(45,84)$ & $62.00(55.5,68.0)$ & 0.64 \\
\hline BMI $\left(\mathrm{kg} / \mathrm{m}^{2}\right)$ & $23.05(20.20,28.06)$ & $23.64(17.93,30.48)$ & $24.98(21.33,25.48)$ & 0.97 \\
\hline \multicolumn{5}{|l|}{ VM } \\
\hline PRT & $1.41(0.58,2.90)$ & $2.03(0.62,25.2)$ & $1.28(0.60,1.68)$ & 0.18 \\
\hline $\mathrm{BRSa}_{1}$ & $48.58(30.34,78.82)$ & $34.05(6.18,128.1)$ & $40.82(40.33,117.61)$ & 0.47 \\
\hline BRSv & $4.20(3.50,6.70)$ & $2.60(1.2,14.3)$ & $3.60(3.30,6.20)$ & 0.14 \\
\hline VR abnormality (\%) & 0 & 29.4 & 0 & 0.12 \\
\hline \multicolumn{5}{|l|}{ Questionnaire } \\
\hline K-COMPASS 31 & $9.00(0,20)$ & $19.00(2,48)$ & $0.00(0,0)$ & 0.00 \\
\hline Ol score & $1.00(0,6)$ & $4.50(0,10)$ & $0.00(0,0)$ & 0.02 \\
\hline
\end{tabular}

Note: Statistically significant values $(p<0.05)$ are highlighted in bold Note: Data are presented as median (range; minimum, maximum). VM, Valsalva maneuver; BMI, body mass index; PRT, pressure recovery time; BRSa, adrenergic barosensitivity; BRSv, cardiovagal barosensitivity; VR, Valsalva ratio; and OI, orthostatic intolerance.

response or the time between the start of the NIRS response and when the hemodynamics returned to the baseline state. For HbT, the area under the curve was calculated during the VM exhalation period using the trapz function in MATLAB ${ }^{\circledR}$. Because $\mathrm{HbT}$ is proportional to blood volume, the integration of this signal can be thought of as sustained blood volume during the VM period. The NIRS parameters were only calculated through the NIRS response obtained from the VM trials suited best for analysis (Fig. 2). The VM trial was selected based on appropriate phase data in the BP measurements. We also used a VM trial that showed an NIRS response with well-defined latency, duration, and amplitude parameters for NIRS analysis.

\subsection{Statistical Analysis}

Patients were divided into two groups: an NVM group and an AbVM group according to their calculated clinical parameters based on VM results. In addition, data for VM and NIRS in healthy subjects were evaluated. Differences in demographic characteristics, questionnaire scores, and proportion of subjects with abnormal results from VM among the patient groups and the normal healthy group were analyzed using either the Kruskal-Wallis test or Fisher's exact test. The NIRS parameters were calculated and compared between the two patient groups with OI and normal healthy subjects using a custom-built script written in MATLAB ${ }^{\circledR}$ (2010A, The MathWorks Inc., Natick, Massachusetts). A significant difference for metrics between the groups was tested using the two-tailed $t$-test. A $p$-value $<0.05$ was considered statistically significant. Pearson's correlation analyses were performed to identify relationships between VM parameters and the NIRS signal.

\section{Results}

\subsection{Subject Characteristics}

Data from 25 patients and 3 normal HCs were analyzed. The patients with OI were subgrouped according to VM findings as follows: (1) NVM ( $n=9$; median age 66.0; 2 males) and (2) AbVM group ( $n=16$; median age 49.0; 9 males). Table 1 presents demographic and clinical characteristics, including VM results, for all subjects.

\subsection{Comparison of NIRS Parameters Between Patients and Healthy Subjects}

NIRS parameters (latency, amplitude, duration, and integration) were calculated for the three subject groups: HC, NVM, and

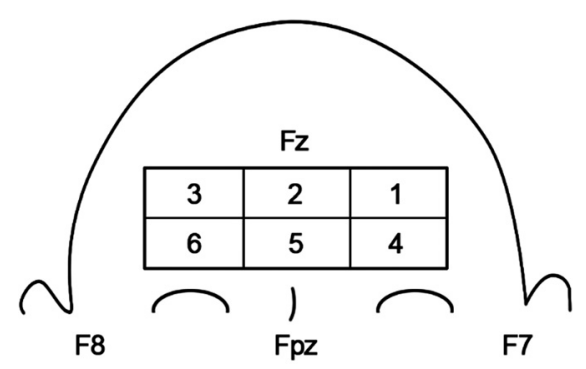

Fig. 3 Probe sectioning used for channel averaging. The NIRS channels were averaged according to six different groups across the forehead. The International $10-10$ coordinates are noted on the diagram for orientation. 
AbVM. Figure 2 shows a sample $\mathrm{BP}$ and $\mathrm{HbO}$ response by (a) $\mathrm{HC}$ and (b) AbVM. In this example of an $\mathrm{HC}$ and AbVM patient, it can be seen that the AbVM had a longer latency and lower amplitude than the $\mathrm{HC}$; while the $\mathrm{HbO}$ peak of the $\mathrm{HC}$ occurred during the phase II period of VM, the peak for the AbVM HbO signal came later, and the slope to peak was also lower compared with the $\mathrm{HC} \mathrm{HbO}$.

We first investigated whether there was evidence of localized hemodynamic changes across the prefrontal area during VM.
The sectioning of the NIRS probe according to its location on the forehead is shown in Fig. 3. The location on the prefrontal area is denoted by the International 10-10 coordinates. Figure 4 shows the box plot for the HbO metrics [(a) latency, (b) amplitude, (c) duration, and (d) HbT integration] for the three groups at a $30-\mathrm{mm}$ SD. It can be seen from the figure that between the six areas, all metrics were measured similarly for the three subject groups. The biggest differences between groups can be seen in the HC group; however, this can be attributed to the low (a)

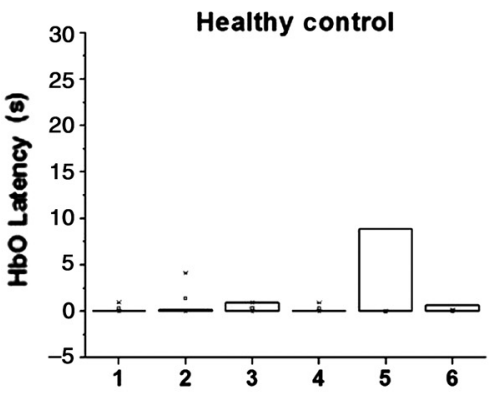

(b)

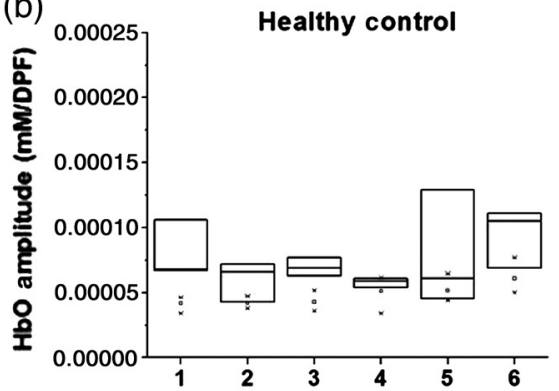

(c)

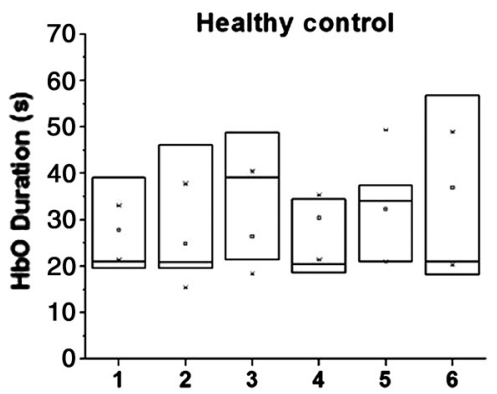

(d)

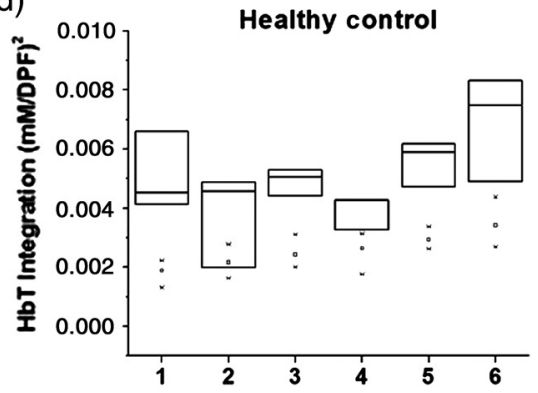

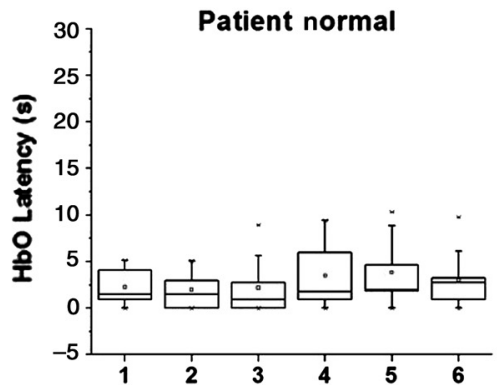
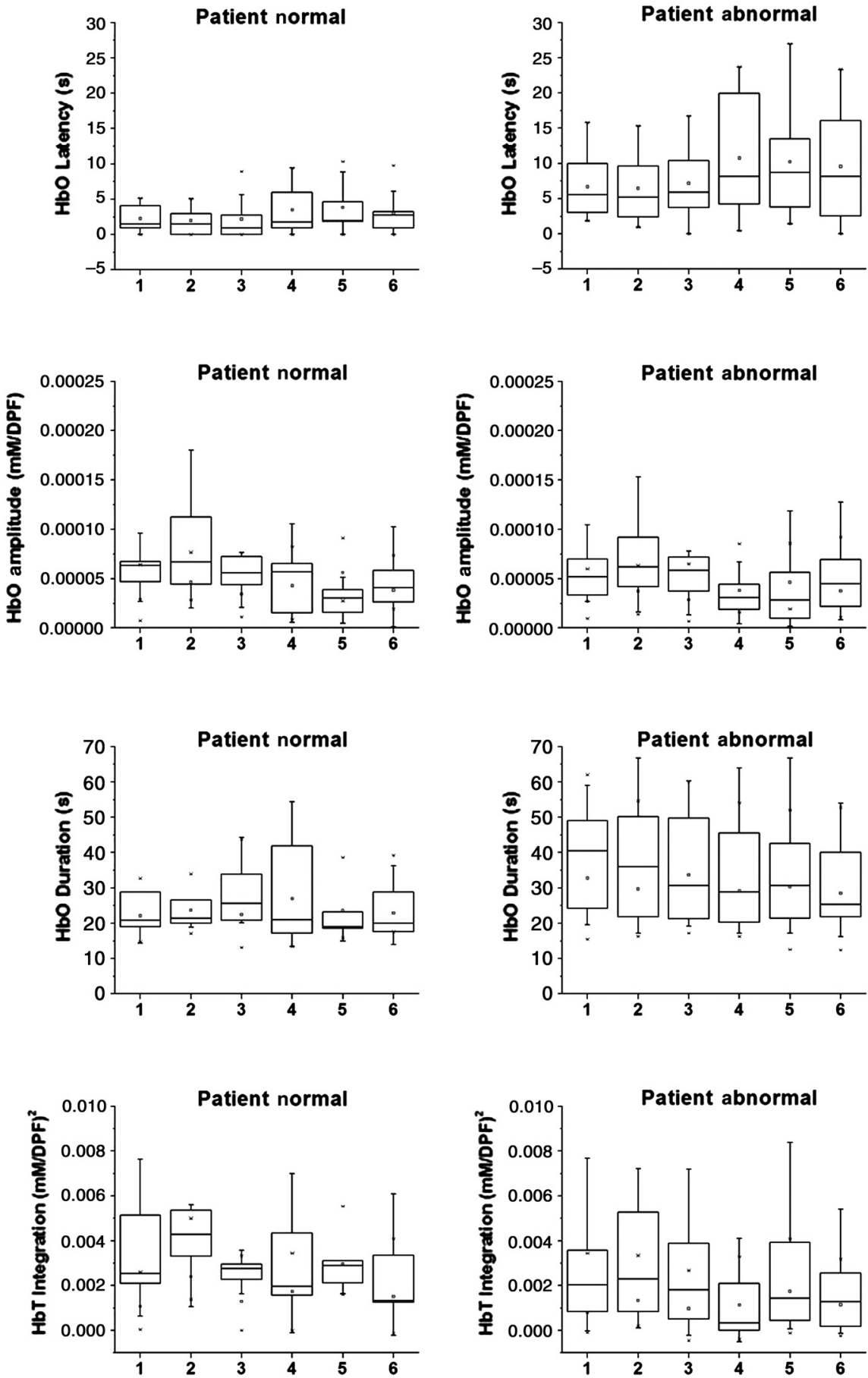

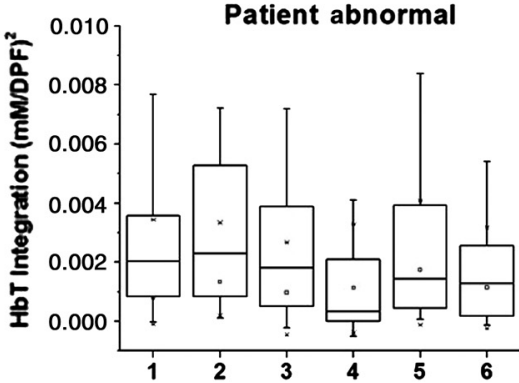

Fig. 4 NIRS metrics for the six regions: (a) HbO latency for 30-mm SD divided by the six regions (as shown in Fig. 3), (b) HbO amplitude for 30-mm SD divided by the six regions, (c) HbO duration for 30-mm $\mathrm{SD}$ divided by the six regions, and (d) HbT integration for 30-mm SD divided by the six regions. 
number of subjects. Thus, for simplicity, the proceeding analysis between groups will be shown for all channels averaged across the prefrontal area.

The average $\mathrm{HbO}$ and $\mathrm{Hb}$ latency for the three groups is shown in Fig. 5. The latencies of the HC and NVM groups were significantly shorter than that of the AbVM group $(p<0.05)$. For the $\mathrm{HC}$ group, the $\mathrm{HbO}$ latency at $30 \mathrm{~mm}$ was $0.39 \pm 0.23 \mathrm{~s}$. The NVM group had an $\mathrm{HbO}$ latency of $2.79 \pm 0.36 \mathrm{~s}$, and the $\mathrm{AbVM}$ group had an $\mathrm{HbO}$ latency of $8.14 \pm 0.55 \mathrm{~s}$. For $\mathrm{Hb}$, the latency at $30 \mathrm{~mm}$ for $\mathrm{HC}, \mathrm{NVM}$, and AbVM was $1.64 \pm 0.51 \mathrm{~s}$, $3.33 \pm 0.41 \mathrm{~s}$, and $5.23 \pm 0.34 \mathrm{~s}$, respectively. Figure 5 shows the statistically significant difference between $\mathrm{Hb}$ latency at the 45-mm distance and shorter channels (i.e., 15 and $30 \mathrm{~mm}$ ). However, the 45-mm distance difference in latency may be attributed to increased scattering of the light reaching that detector. In addition, there were no significant differences between SDs by latency. Overall, our results showed that OI patients had a longer latency for onset of the hemodynamic response than the $\mathrm{HC}$ group, with the AbVM group having the longest latency.

While latency clearly distinguished between the groups, amplitude and duration were not significantly different between groups for the NIRS response. The full list of $p$-values between groups for NIRS metrics obtained with a two-tailed $t$-test is presented in Table 2. Although there was no statistical significance, certain patterns can be observed in Figs. 6 and 7. The amplitude of the $\mathrm{HbO}$ and $\mathrm{Hb}$ response tended to peak at the shorter separations for all groups and decreased as channel distance became longer, as shown in Fig. 6. The drop-off in amplitude is especially noticeable in the $\mathrm{Hb}$ response. For amplitude, there was a statistical difference at the $15-\mathrm{mm}$ distance and all other distances for all groups in their $\mathrm{HbO}$ and $\mathrm{Hb}$ responses. For farther channels, the number of distances that were statistically different was reduced, showing that amplitude was drastically reduced with longer channels.

In terms of duration, Fig. 7 shows that $\mathrm{HbO}$ and $\mathrm{Hb}$ duration did not show any significant pattern between the groups. The

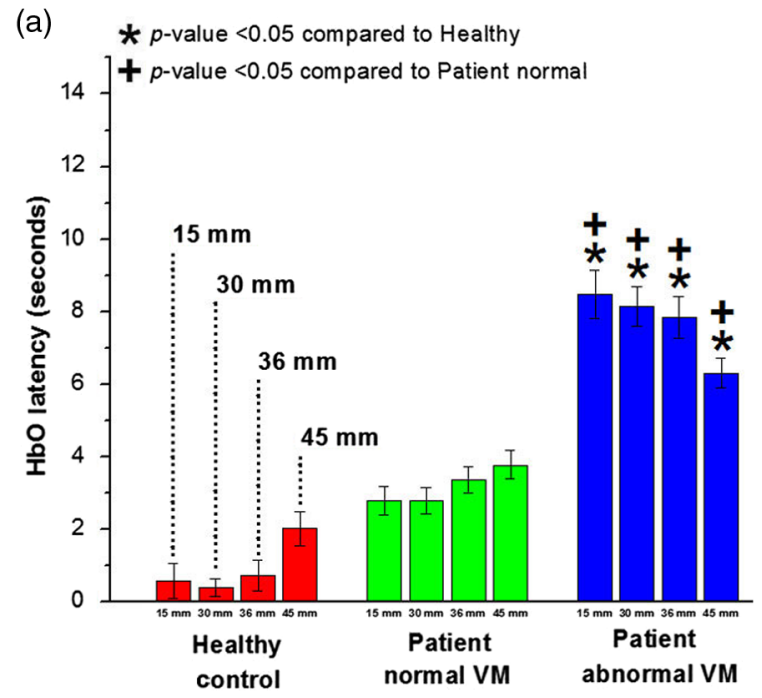

difference between channel distances within a group did not demonstrate any recurring trend; however, the 45-mm channels did show the longest duration in the $\mathrm{Hb}$ signal for the healthy group.

As a measure of sustained blood volume in the prefrontal area during $\mathrm{VM}, \mathrm{HbT}$ integration was compared among the three groups (Fig. 8). The HbT integration was significantly different between the $\mathrm{HC}$ and $\mathrm{AbVM}$ groups. The $\mathrm{HC}$ group showed a higher sustained blood volume change in the prefrontal superficial layer during VM compared with the OI groups. Similar to the amplitude metric, the shortest channels were significantly different from other distances for all groups, and the number of statistically significant distances was reduced as the channels became farther apart.

To observe the effect of superficial channels, the shorter channels $(15 \mathrm{~mm}$ ) were regressed from 30,36 , and $45 \mathrm{~mm}$ data, using previously described procedures. ${ }^{15}$ Figure 9 shows the $\mathrm{HbO}$ metrics recalculated after short channel removal. At $30 \mathrm{~mm}$, latency was significantly different $(p<0.05)$ between healthy and NVM/AbVM [Fig. 9(a)]. In addition, once the short channels were regressed, $\mathrm{HbO}$ duration was significantly different between the healthy and OI patients at $30 \mathrm{~mm}$ [Fig. 9(c)]. However, there was no significant difference between NVM and $\mathrm{AbVM}$ in the $\mathrm{HbO}$ and $\mathrm{HbT}$ integration signal when the short channel was regressed.

\subsection{Correlation of NIRS Parameters to VM Parameters}

We also investigated whether there was any relationship between our derived NIRS parameter and the typical VM parameters used to diagnosis clinical symptoms. We performed a linear regression for the NIRS parameters ( $\mathrm{HbO}, \mathrm{Hb}$ : latency, duration, amplitude, and HbT integration) averaged across the entire prefrontal area, per SD, and the VM parameters (VR, PRT, $\mathrm{BRSa}_{1}$, and $\mathrm{BRSv}$ ). According to our regression analysis, the only combination of NIRS and VM parameters with a

(b)

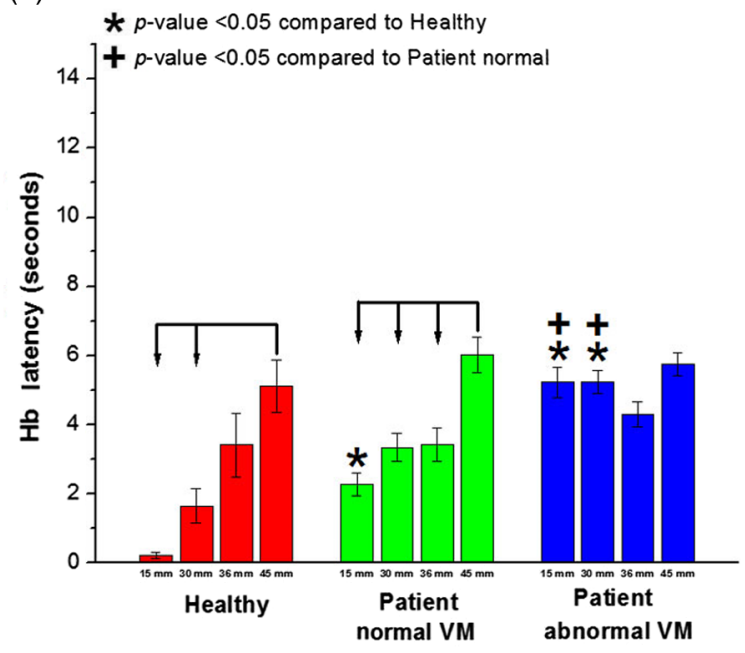

Fig. 5 Latency for all averaged channels: (a) HbO latency and (b) $\mathrm{Hb}$ latency for all channels are averaged according to source-detector separation. The bars are ordered from left to right from shortest separation $(15 \mathrm{~mm})$ to farthest separation $(45 \mathrm{~mm})$. Healthy subjects showed the smallest latency at the shorter distance source-detector separations, especially for the $\mathrm{HbO}$ signal. Statistically significant values $(p<0.5)$ compared with the healthy group or patient NVM group are depicted by * or + , respectively. Statistically significant values $(p<0.5)$ between distances within a group are connected via arrows. 
Kim et al.: Prefrontal hemodynamic changes measured using near-infrared spectroscopy during the Valsalva maneuver in patients...

Table $2 p$-values from a two-tailed $t$-test for groups of patients (healthy, patient normal, and patient abnormal) for each metric (latency, amplitude, duration, and $\mathrm{HbT}$ integration) at different hemodynamic measurements ( $\mathrm{HbO}, \mathrm{Hb}$, and $\mathrm{HbT}$ ).

\begin{tabular}{|c|c|c|c|c|}
\hline & $15 \mathrm{~mm}$ & $30 \mathrm{~mm}$ & $36 \mathrm{~mm}$ & $45 \mathrm{~mm}$ \\
\hline \multicolumn{5}{|l|}{$\mathrm{HbO}$} \\
\hline & \multicolumn{4}{|c|}{ Latency } \\
\hline Healthy/patient normal & 0.182 & 0.118 & 0.108 & 0.176 \\
\hline Healthy/patient abnormal & 0.011 & 0.013 & 0.012 & 0.018 \\
\hline \multirow[t]{2}{*}{ Patient normal/patient abnormal } & 0.003 & 0.004 & 0.008 & 0.023 \\
\hline & \multicolumn{4}{|c|}{ Amplitude } \\
\hline Healthy/patient normal & 0.413 & 0.481 & 0.149 & 0.372 \\
\hline Healthy/patient abnormal & 0.272 & 0.234 & 0.180 & 0.572 \\
\hline \multirow[t]{2}{*}{ Patient normal/patient abnormal } & 0.791 & 0.458 & 0.686 & 0.703 \\
\hline & \multicolumn{4}{|c|}{ Duration } \\
\hline Healthy/patient normal & 0.669 & 0.250 & 0.071 & 0.810 \\
\hline Healthy/patient abnormal & 0.515 & 0.891 & 0.684 & 0.787 \\
\hline Patient normal/patient abnormal & 0.102 & 0.091 & 0.105 & 0.462 \\
\hline
\end{tabular}

$\mathrm{Hb}$

\begin{tabular}{llllr}
\hline & & \multicolumn{3}{c}{ Latency } \\
\hline Healthy/patient normal & $\mathbf{0 . 0 4 1 6}$ & 0.1431 & 0.9976 & 0.4696 \\
Healthy/patient abnormal & $\mathbf{0 . 0 2 1 6}$ & $\mathbf{0 . 0 1 6 0}$ & 0.5790 & 0.5914 \\
Patient normal/patient abnormal & $\mathbf{0 . 0 2 0 2}$ & $\mathbf{0 . 0 3 7 9}$ & 0.4025 & 0.7288 \\
\hline
\end{tabular}

\begin{tabular}{|c|c|c|c|c|}
\hline & \multicolumn{4}{|c|}{ Amplitude } \\
\hline Healthy/patient normal & 0.494 & 0.746 & 0.615 & 0.827 \\
\hline Healthy/patient abnormal & 0.320 & 0.702 & 0.698 & 0.593 \\
\hline \multirow[t]{2}{*}{ Patient normal/patient abnormal } & 0.543 & 0.840 & 0.771 & 0.274 \\
\hline & \multicolumn{4}{|c|}{ Duration } \\
\hline Healthy/patient normal & 0.177 & 0.083 & 0.889 & 0.236 \\
\hline Healthy/patient abnormal & 0.145 & 0.082 & 0.235 & 0.469 \\
\hline Patient normal/patient abnormal & 0.429 & 0.392 & 0.063 & 0.499 \\
\hline \multicolumn{5}{|l|}{$\mathrm{HbT}$} \\
\hline & \multicolumn{4}{|c|}{ Integration } \\
\hline Healthy/patient normal & 0.243 & 0.239 & 0.181 & 0.305 \\
\hline Healthy/patient abnormal & 0.030 & 0.026 & 0.026 & 0.102 \\
\hline Patient normal/patient abnormal & 0.230 & 0.129 & 0.151 & 0.422 \\
\hline
\end{tabular}

Note: Statistically significant values $(p<0.05)$ are highlighted in bold. 

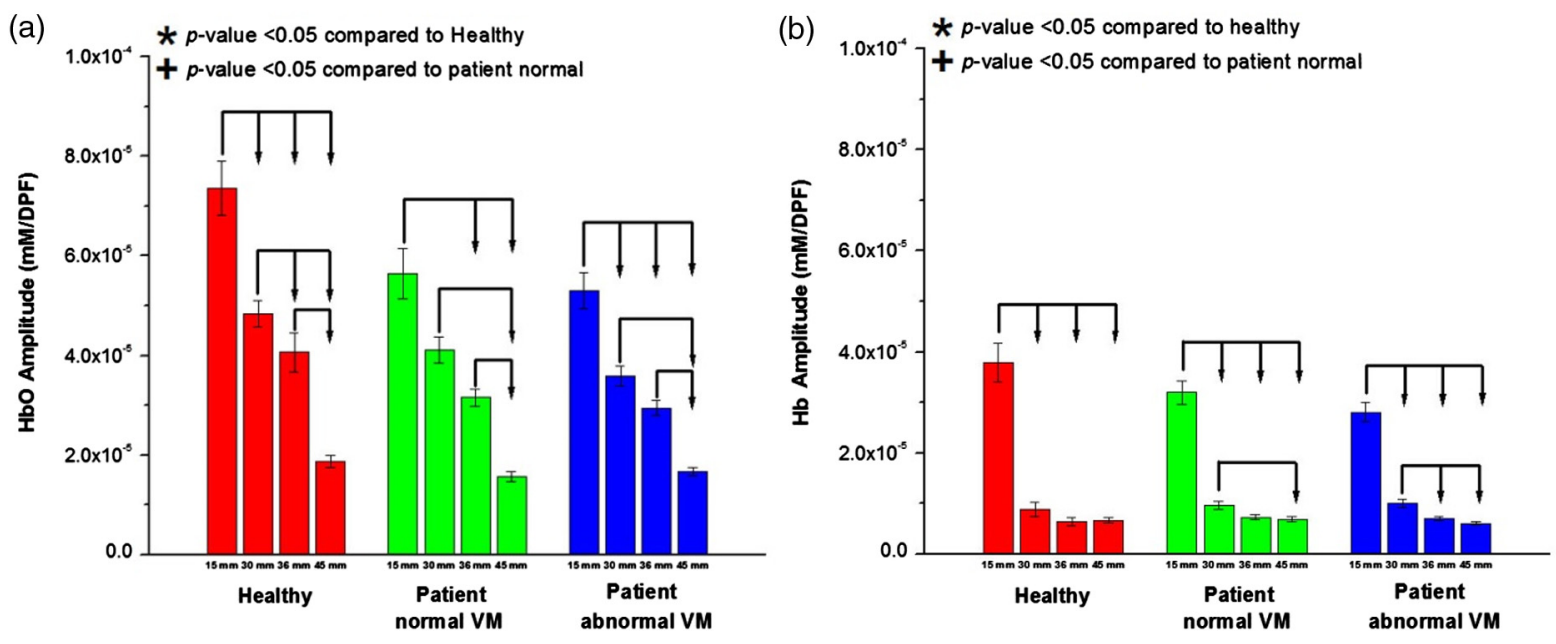

Fig. 6 Amplitude for all channels averaged according to source-detector separation. The amplitude of (a) $\mathrm{HbO}$ and (b) $\mathrm{Hb}$ changes peaked at shorter channels for all patient groups and dropped for the longer channels. Statistically significant values $(p<0.5)$ compared with the healthy group or patient NVM group are depicted by * or + , respectively. Statistically significant values $(p<0.5)$ between distances within a group are connected via arrows.
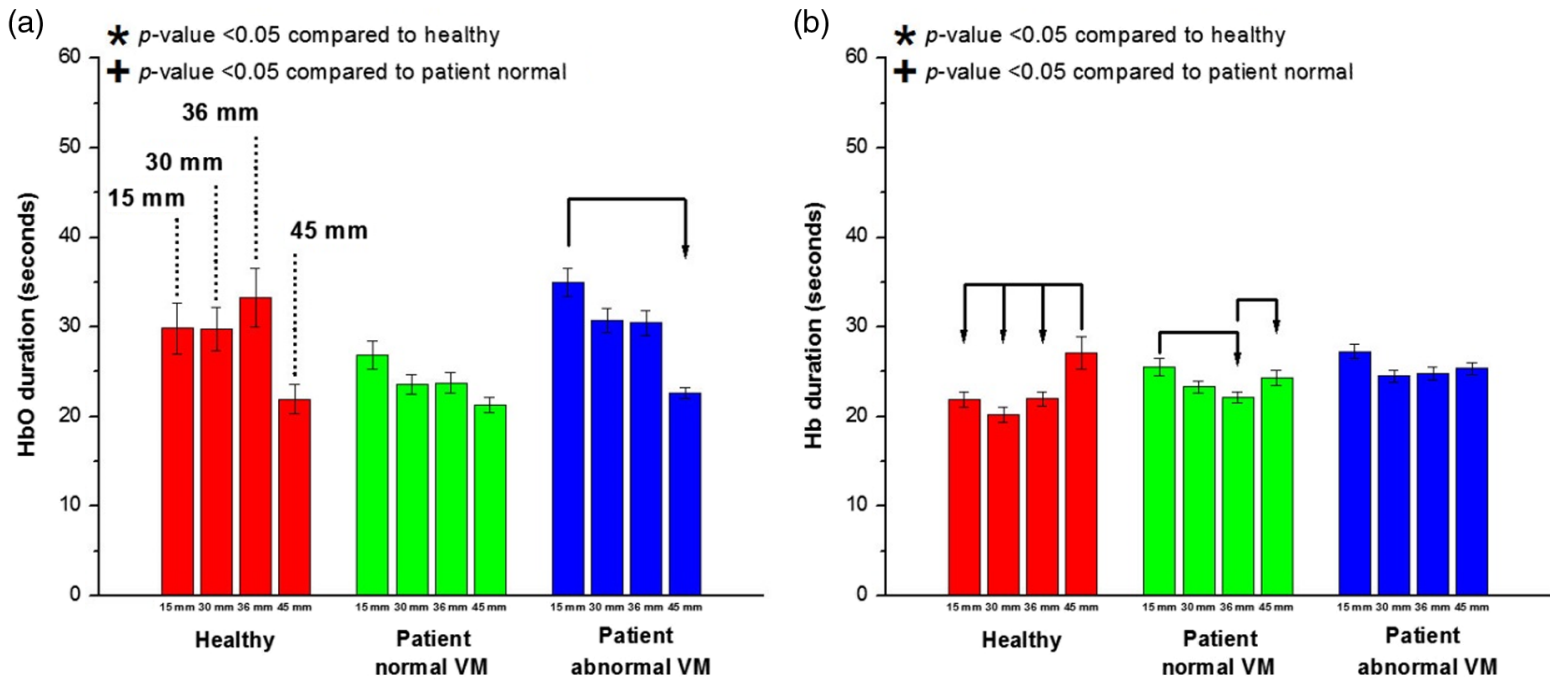

Fig. 7 Duration for all channels averaged according to source-detector separation. The bars are ordered from left to right from shortest separation $(15 \mathrm{~mm})$ to farthest separation $(45 \mathrm{~mm})$ for the recorded NIRS duration of (a) $\mathrm{HbO}$ and (b) $\mathrm{Hb}$. The duration parameter shows less difference among the groups. Statistically significant values $(p<0.5)$ compared with the healthy group or patient NVM group are depicted by * or + , respectively. Statistically significant values $(p<0.5)$ between distances within a group are connected via arrows.

statistically significant relationship was $\mathrm{HbO}$ duration at $15-\mathrm{mm}$ source-detector separation and VR $\left(R^{2}=0.164, p=0.032\right)$. The results of the linear regression are shown in Fig. 10. When $\mathrm{HbO}$ duration at $15 \mathrm{~mm}$ and VR were tested for each group, there was no significant relationship between the parameters. Results of the linear regression per group were as follows: $\mathrm{HC}\left(R^{2}=0.003, p=0.964\right), \mathrm{NVM}\left(R^{2}=0.159, p=0.288\right)$, and $\operatorname{AbVM}\left(R^{2}=0.207, p=0.076\right)$. The small $\mathrm{HC}$ size and similar values in VR between NVM (average: $1.58 \pm 0.28$ ) and AbVM (average: $1.62 \pm 0.35$ ) resulted in a poor fit for each group individually. However, when all the subjects were grouped together, the fit was statistically significant.

\section{Discussion}

VM performance can be measured by various clinical parameters using BP and HR data. Baroreceptor sensitivity has been represented through parameters such as $\mathrm{BRSv}$ and $\mathrm{BRSa}_{1}{ }^{27}$ In addition, we measured VR and PRT for all subjects. VR is the most commonly used parameter to estimate cardiovagal function. However, VR is often not sensitive enough to accurately detect patient abnormal cardiovagal function; ${ }^{36}$ therefore, other clinical parameters should also be considered to discriminate abnormal cardiovagal function. The symptom questionnaire showed significantly higher scores in the patient group 


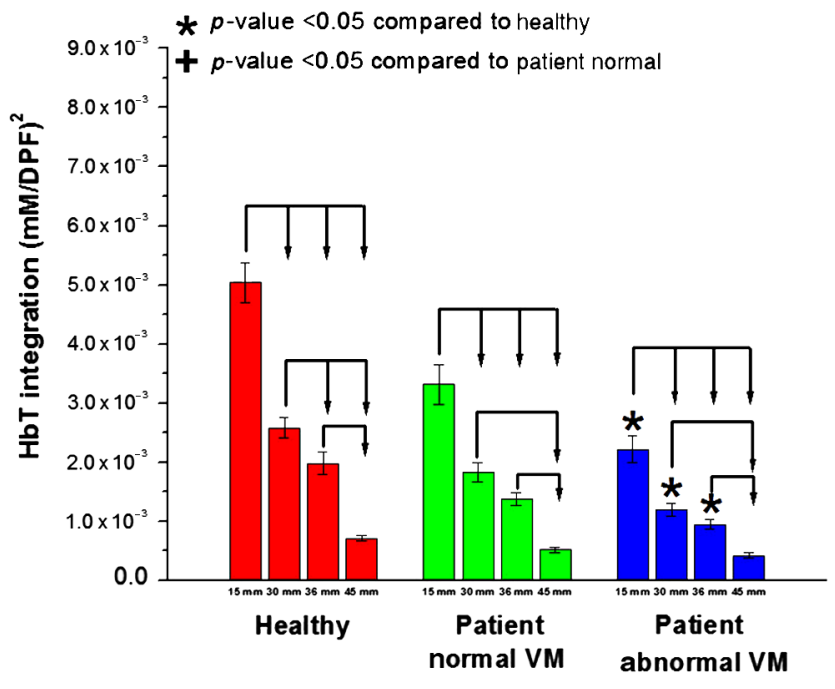

Fig. $8 \mathrm{HbT}$ integration for all channels averaged according to sourcedetector separation. The bars are ordered from left to right from shortest separation $(15 \mathrm{~mm})$ to farthest separation $(45 \mathrm{~mm})$ for the integration of the $\mathrm{HbT}$ signal. Statistically significant values $(p<0.5)$ compared with the healthy group or patient NVM group are depicted by * or + , respectively. Statistically significant values $(p<0.5)$ between distances within a group are connected via arrows.

compared with the HC group and even higher in patients with AbVM compared with patients with NVM. This suggests that discrimination of VM abnormality is best achieved through multiple parameters to be reliable. In addition to clinical parameters, we analyzed VM performance between groups with NIRS to monitor relative $\mathrm{HbO}, \mathrm{Hb}$, and $\mathrm{HbT}$ concentration changes. We analyzed the latency, amplitude, duration, and HbT integration (i.e., sustained blood volume) of the NIRS response to distinguish between HC, NVM, and AbVM during VM.

Of these parameters, latency showed the highest contrast among the groups. The AbVM group had longer latency for $\mathrm{HbO}$ changes for all source-detector separations compared with NVM $(p<0.05)$. Cerebral perfusion pressure is defined as MAP minus intracranial pressure (ICP) or MAP minus central venous pressure (CVP), if CVP > ICP. VM leads to a proportional elevation in CVP, a marked reduction in cardiac output, and characteristic changes in MAP. ${ }^{37-39}$ In particular, the abrupt reduction in MAP challenges the regulation of cerebral perfusion and can result in syncope. ${ }^{40}$ Our hypothesis is that increased CVP leads to sustained blood volume retention in the superficially distributed venous system of the forehead, which leads to rapid detection of cerebral hemodynamics in a shallow channel and a short latency for the NIRS signal. We believe that, in the $\mathrm{AbVM}$, the latency can be increased due to the interruption of hemodynamic autoregulation compared with NVM. AbVM also showed a pattern of more diminished $\mathrm{HbO}$ response amplitude compared with NVM, with a reduced response as the SD increased, except in the 45-mm channel; however, the results between groups were not statistically significant, but the difference between distances was significant. Compared with AbVM, the NVM did not differ statistically in sustained volume, but the $\mathrm{HC}$ showed a difference during VM except for the farthest channel (Fig. 8). The lack of significant differences in the 45-mm channel for the NIRS metrics may be attributed to strong light scattering.

The calculated NIRS metrics can provide clinical insight into cerebral perfusion during VM. Delayed HbO latency may represent delayed cerebral perfusion in patients with AbVM compared with patients with NVM. Although statistical significance was not shown, even OI patients with NVM results showed slightly delayed $\mathrm{HbO}$ compared with $\mathrm{HC}$. According to the 2017 ACC/AHA/HRS guideline for the evaluation and management of patients with syncope, the mechanism of syncope, one of the symptoms of OI, is explained by cerebral hypoperfusion. ${ }^{41}$ Lankford et al. showed that hypotension associated with OI leads to decreased cerebral perfusion. This decreased perfusion is working as a cause for neurologic clinical manifestations. ${ }^{13}$ Gulli et al. stated that patients suffering from poor OI fail to maintain an adequate brain perfusion pressure. This study showed that prolonged delay in the baroreflex response was associated with the poor OI. ${ }^{42}$ These findings underlie suspicion that OI symptoms might develop due to delayed cerebral perfusion, which might cause an oxygen deficit for a few seconds.

To assess the overall recruitment of cerebral hemodynamics during the VM, we evaluated the amplitude of the NIRS signal; there was no difference in $\mathrm{HbO}$ amplitude by group, but there was a difference according to NIRS channel distance. While $\mathrm{HbO}$ amplitude showed a pattern among the groups, Hb amplitude did not show any pattern except for the 15-mm SD distance. In general, neuronal activities in normal subjects cause a decrease in $\mathrm{Hb}$ associated with increases in $\mathrm{HbO}$ and $\mathrm{HbT}$ in the activated cortical area caused by $\mathrm{O}_{2}$ delivery in excess of $\mathrm{O}_{2}$ consumption. ${ }^{43,44}$ However, the brain dysfunction may present various forms of $\mathrm{Hb}$ metabolism during neuronal activation and the NIRS signal in a short source-detector channel (e.g., $15 \mathrm{~mm}$ ) may be affected by sinus and/or forehead skin vasculature, so there may not be any difference in $\mathrm{Hb}$ response. ${ }^{32,45}$

The HbT integration, which is related to cerebral blood volume, ${ }^{46}$ was not significantly different between OI groups. The $\mathrm{HC}$ group showed higher sustained blood volume changes in the prefrontal superficial layer during VM compared with the OI groups. VM leads to a proportional elevation in intrathoracic and CVP. The increase in CVP causes retention of sustained blood volume in the venous system of the forehead and causes superficial blood volume changes. We observed an increased $\mathrm{HbT}$ in the mainly $\mathrm{HC}$ group and the patients with NVM group compared with patients with AbVM group. The reason for the increased $\mathrm{HbT}$ in mainly shallow channels over other channels is believed to reflect the venous and/or sinus system and skin vascular pooling occurring at the measurement sites. VM produces a marked increase in blood volume in extracranial compartments. The increased extracranial blood volume heavily affects HbT parameters. This may reflect skin vascular pooling, since extracranial signals may be present in these NIRS signals. ${ }^{9,47}$ A study showed that the average scalp thickness was $6.9 \pm 3.6 \mathrm{~mm}$, while the average skull thickness was $6.0 \pm$ $1.9 \mathrm{~mm} .{ }^{48}$ Considering the important effects of overlying scalp and skull layers on the measurement of brain hemodynamics, the reason that the short $\mathrm{SD}(15 \mathrm{~mm})$ is consistently showing a significant difference in NIRS analysis during VM may be due to general superficial hemodynamics influenced during VM, rather than cerebral hemodynamics. Moreover, once the short channels were regressed, HbO latency was significantly different between the groups.

Consequently, HbT integration, along with duration and amplitude, did not show as large a contrast between the NVM and AbVM groups as latency did; thus, we concluded that latency had the most potential as a quality metric for identifying an AbVM response in patients with OI. 

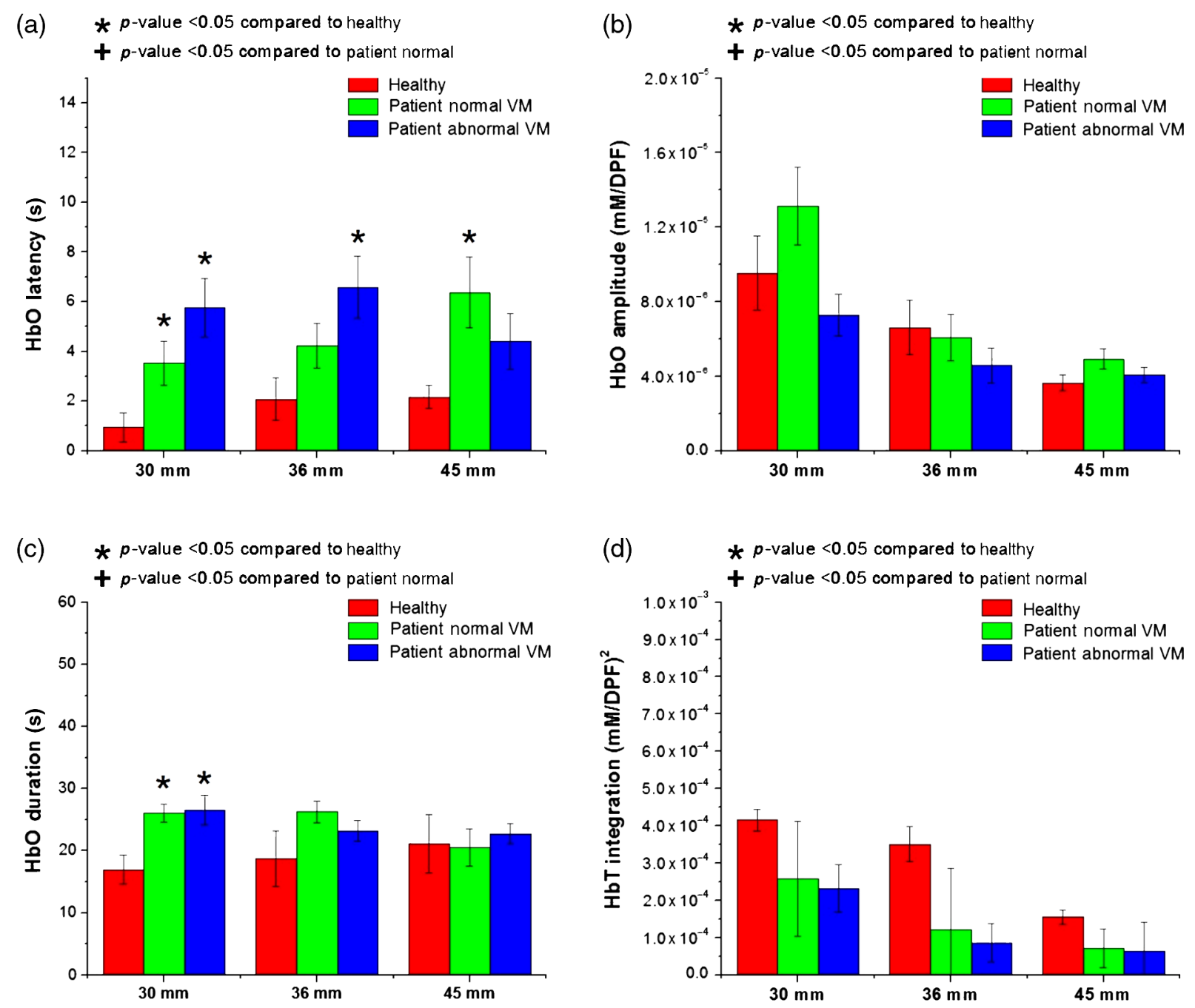

Fig. 9 Calculated metrics with short channel removed: (a) $\mathrm{HbO}$ latency, (b) amplitude, (c) duration, and (d) $\mathrm{HbT}$ integration calculated for 30,36 , and $45 \mathrm{~mm}$, with the $15-\mathrm{mm}$ channel regressed from the data. Statistically significant values $(p<0.5)$ compared with the healthy group or patient normal group are depicted as a * or + , respectively.

This study had some limitations. First, there were no data related to cerebrovascular status in our subjects. We tried to exclude any possibility of CNS abnormality, including cerebrovascular abnormality, via a thorough medical interview and neurologic examination. Further studies with cerebral vascular evaluation, such as MR angiography, would provide more reliable scientific results. The other limitation is the small $\mathrm{HC}$ sample size. There was no statistically significant different response for latency, but this may be attributed to the limited HC group sample size. Although the number of HCs may be seen as a limitation of this study, we believe that the dataset is reliable. We rigorously enforced inclusion criteria for the $15 \mathrm{HC}$ subjects. The criteria excluded subjects with any problem that showed up in the OI questionnaires and subjects who showed AbVM data or potential signs of disorders of the ANS or CNS. After the screening, we were left with three HCs who were able to pass multiple OI tests [i.e., questionnaires, VM, etc. (Table 1)]. Because of this strict screening, we believe that our HC subject data represent subjects with no signs of OI.

An additional limitation may result from the distances of the NIRS probe sources and detectors. With multiple source- detector analysis, shorter channel regressions are typically performed to isolate the hemodynamic response from the superficial area and the cerebral response. Further investigation in this area would be worthwhile, but it has been shown that VM is largely a systemic maneuver whose signal comes predominantly from superficial hemodynamic changes rather than cerebral changes. ${ }^{15}$ Previous studies have shown that the ideal distance to separate the superficial changes is $\sim 8 \mathrm{~mm} .{ }^{49}$ However, considering the thickness of the overlying scalp and skull layers, the suitable distance to measure cerebral activity is between 20 - and 45-mm SD separations. ${ }^{48}$ For this reason, although the shortest SD distance was $15 \mathrm{~mm}$ due to the design of our NIRS probe, it may be a reasonable distance to observe superficial changes.

Although Fig. 10 shows a correlation between NIRS parameters ( $\mathrm{HbO}$ duration) and the clinical VM parameter (VR), it is worth investigating the intertwined relationships between our derived NIRS parameters and the clinically used VM parameters. Our current study focused on the prefrontal hemodynamic changes rather than the physiological significance and relationship between the two parameter groups (NIRS and VM). By closely understanding how cerebral perfusion and autoregulatory systems 


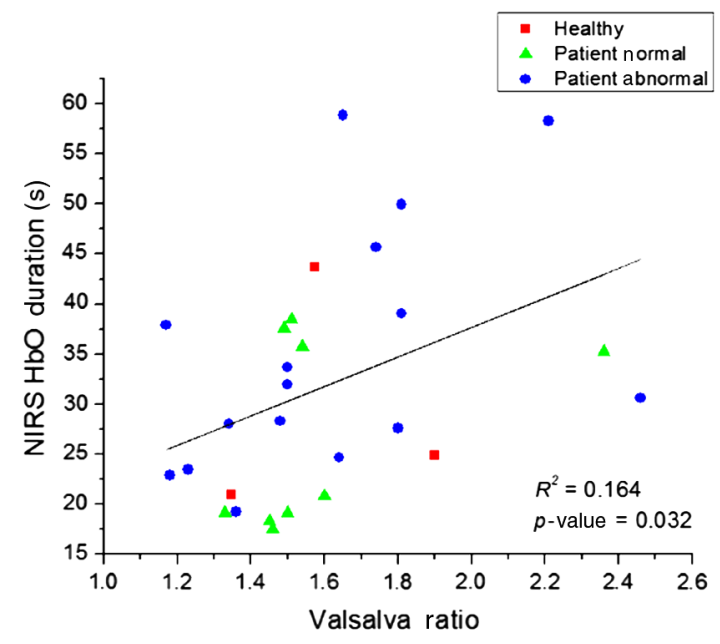

Fig. 10 Linear regression analysis of NIRS and VM parameters. Each patient group is designated by a specific shape. This combination of $\mathrm{HbO}$ duration (15-mm source-detector separation) and VR was the only combination that showed a statistically significant difference $(p<0.05)$.

affect these metrics during VM, we can better quantify and analyze the cerebral autoregulatory system.

NIRS offers an alternate solution for understanding the effects of a dysregulated ANS on the delivery of blood to the brain. In addition, NIRS has the potential to monitor patients with inconclusive BP or HR readings. Our results confirm the presence of a delay in hemodynamic response according to the VM procedure. Although the delay in the recruitment of hemodynamics to the frontal area during VM is still largely unknown, ${ }^{50}$ differentiating between healthy subjects and OI patients according to VM performance is important for understanding the autoregulatory nature of the nervous system. For further study of cerebral hemodynamics during VM, larger sample sizes are required, including normal healthy participants, and detailed studies should be conducted at deeper layers during NIRS.

\section{Disclosures}

All of the authors had no relevant financial or competing interests and no other potential conflicts of interest.

\section{Acknowledgments}

This research was supported by the Original Technology Research Program for Brain Science through the National Research Foundation (NRF) of Korea funded by the Ministry of Science ICT and Future Planning (MSIP) (No. 2017M3C7A1048566) and the NRF of Korea Grant funded by the Korean Government (MSIP) (No. NRF2015R1A5A7037674). The funders had no role in study design, data collection and analysis, decision to publish, or preparation of the paper.

\section{References}

1. P. Novak, "Quantitative autonomic testing," J. Visualized Exp. (53), e2502 (2011).

2. P. A. Low, "Autonomic nervous system function," J. Clin. Neurophysiol. 10(1), 14-27 (1993).

3. P. A. Low and V. A. Tomalia, "Orthostatic hypotension: mechanisms, causes, management," J. Clin. Neurol. 11(3), 220-226 (2015).
4. G. Tian et al., "External counterpulsation reduces beat-to-beat blood pressure variability when augmenting blood pressure and cerebral blood flow in ischemic stroke," J. Clin. Neurol. 12(3), 308-315 (2016).

5. W. Wendling et al., "Cardiovascular and cerebrovascular effects of the applied Valsalva manoeuvre in anaesthetized neurosurgical patients," Eur. J. Anaesthesiol. 11(2), 81-87 (1994).

6. G. Nath, G. Korula, and M. J. Chandy, "Effect of intrathecal saline injection and Valsalva maneuver on cerebral perfusion pressure during transsphenoidal surgery for pituitary macroadenoma," J. Neurosurg. Anesthesiol. 7(1), 1-6 (1995).

7. G. Ossard et al., "Cerebral blood flow velocity response induced by a $70-\mathrm{hPa}$ Valsalva manoeuvre associated with normo- and hypergravity in humans," Eur. J. Appl. Physiol. Occup. Physiol. 72(5-6), 502-508 (1996).

8. F. Pott et al., "Middle cerebral artery blood velocity during a Valsalva maneuver in the standing position," J. Appl. Physiol. 88(5), 1545-1550 (2000).

9. B. G. Perry et al., "Cerebral hemodynamics during graded Valsalva maneuvers," Front. Physiol. 5, 349 (2014).

10. B. Wang et al., "Posture-related changes in brain functional connectivity as assessed by wavelet phase coherence of NIRS signals in elderly subjects," Behav. Brain Res. 312, 238-245 (2016).

11. M. A. Yucel et al., "Short separation regression improves statistical significance and better localizes the hemodynamic response obtained by near-infrared spectroscopy for tasks with differing autonomic responses," Neurophotonics 2(3), 035005 (2015).

12. K. J. Stone et al., "The validity and reliability of continuous-wave nearinfrared spectroscopy for the assessment of leg blood volume during an orthostatic challenge," Atherosclerosis 251, 234-239 (2016).

13. J. Lankford et al., "Cerebral blood flow during HUTT in young patients with orthostatic intolerance," Clin. Auton. Res. 25(5), 277-284 (2015).

14. A. Tsubaki et al., "Effect of Valsalva maneuver-induced hemodynamic changes on brain near-infrared spectroscopy measurements," Oxygen Transport to Tissue XXXV, Vol. 789, pp. 97-103, Springer, New York (2013).

15. R. Saager and A. Berger, "Measurement of layer-like hemodynamic trends in scalp and cortex: implications for physiological baseline suppression in functional near-infrared spectroscopy," J. Biomed. Opt. 13(3), 034017 (2008).

16. F. Pott et al., "Middle cerebral artery blood velocity during intense static exercise is dominated by a Valsalva maneuver," J. Appl. Physiol. 94(4), 1335-1344 (2003).

17. M. Laures et al., "Assessing the role of cerebral autoregulation during intrathoracic pressure changes by near infrared spectroscopy (NIRS)," Eur. Respir. J. 46(59), PA2355 (2015).

18. A. H. van Beek et al., "Cerebral autoregulation: an overview of current concepts and methodology with special focus on the elderly," J. Cereb. Blood Flow Metab. 28(6), 1071-1085 (2008).

19. P. A. Low et al., "Prospective evaluation of clinical characteristics of orthostatic hypotension," Mayo Clin. Proc. 70(7), 617-622 (1995).

20. J. B. Kim et al., "Utility of corrected QT interval in orthostatic intolerance," PLoS One 9(9), e106417 (2014).

21. D. M. Sletten et al., "COMPASS 31: a refined and abbreviated composite autonomic symptom score," Mayo Clin. Proc. 87(12), 1196-1201 (2012).

22. J. B. Kim et al., "Autonomic dysfunction according to disease progression in Parkinson's disease," Parkinsonism Relat. Disord. 20(3), 303307 (2014).

23. E. R. Vogel, P. Sandroni, and P. A. Low, "Blood pressure recovery from Valsalva maneuver in patients with autonomic failure," Neurology 65(10), 1533-1537 (2005).

24. C. C. Huang et al., "Effect of age on adrenergic and vagal baroreflex sensitivity in normal subjects," Muscle Nerve 36(5), 637-642 (2007).

25. I. Palamarchuk et al., "Baroreflex sensitivity: reliability of baroreflex components of the Valsalva maneuver," Auton. Neurosci. 185, 138140 (2014).

26. S. Roy et al., "Cardiovagal baroreflex sensitivity in Parkinson's disease and multiple-system atrophy," J. Clin. Neurol. 12(2), 218-223 (2016).

27. C. Schrezenmaier et al., "Adrenergic and vagal baroreflex sensitivity in autonomic failure," Arch. Neurol. 64(3), 381-386 (2007).

28. T. Goto et al., "Lateralized frontal activity for Japanese phonological processing during child development," Front. Hum. Neurosci. 9, 417 (2015). 
29. Y. Kita et al., "Self-face recognition in children with autism spectrum disorders: a near-infrared spectroscopy study," Brain Dev. 33(6), 494-503 (2011)

30. E. Okada et al., "Theoretical and experimental investigation of nearinfrared light propagation in a model of the adult head," Appl. Opt. 36(1), 21-31 (1997).

31. G. Strangman, M. A. Franceschini, and D. A. Boas, "Factors affecting the accuracy of near-infrared spectroscopy concentration calculations for focal changes in oxygenation parameters," NeuroImage 18(4), 865-879 (2003).

32. K. Sakatani et al., "Cerebral blood oxygenation changes induced by auditory stimulation in newborn infants measured by near infrared spectroscopy," Early Hum. Dev. 55(3), 229-236 (1999).

33. H. Liu et al., "Noninvasive investigation of blood oxygenation dynamics of tumors by near-infrared spectroscopy," Appl. Opt. 39(28), 5231-5243 (2000).

34. S. Prahl, Optical Absorption of Hemoglobin, Oregon Medical Laser Center (1999).

35. A. Villringer and B. Chance, "Non-invasive optical spectroscopy and imaging of human brain function," Trends Neurosci. 20(10), 435442 (1997).

36. A. H. Rothschild et al., "Sensitivity of R-R variation and Valsalva ratio in assessment of cardiovascular diabetic autonomic neuropathy," Diabetes Care 10(6), 735-741 (1987).

37. H. Goldberg, E. I. Elisberg, and L. N. Katz, "The effects of the Valsalvalike maneuver upon the circulation in normal individuals and patients with mitral stenosis," Circulation 5(1), 38-47 (1952).

38. F. P. Tiecks et al., "Effects of the Valsalva maneuver on cerebral circulation in healthy adults. A transcranial Doppler study," Stroke 26(8), 1386-1392 (1995).

39. F. Pott et al., "Middle cerebral artery blood velocity during a Valsalva maneuver in the standing position," J. Appl. Physiol. 88(5), 1545-1550 (2000).

40. R. C. Duvoisin, "The Valsalva maneuver in the study of syncope," Electroencephalogr. Clin. Neurophysiol. 13, 622-626 (1961).

41. W. K. Shen et al., "2017 ACC/AHA/HRS guideline for the evaluation and management of patients with syncope: a report of the American
College of Cardiology/American Heart Association Task Force on Clinical Practice Guidelines and the Heart Rhythm Society," Heart Rhythm 14(8), e155-e217 (2017).

42. G. Gulli et al., "Prolonged latency in the baroreflex mediated vascular resistance response in subjects with postural related syncope," Clin. Auton. Res. 15(3), 207-212 (2005).

43. C. Hock et al., "Age dependency of changes in cerebral hemoglobin oxygenation during brain activation: a near-infrared spectroscopy study," J. Cereb. Blood Flow Metab. 15(6), 1103-1108 (1995).

44. C. Hock et al., "Decrease in parietal cerebral hemoglobin oxygenation during performance of a verbal fluency task in patients with Alzheimer's disease monitored by means of near-infrared spectroscopy (NIRS)-correlation with simultaneous rCBF-PET measurements," Brain Res. 755(2), 293-303 (1997).

45. K. Sakatani et al., "Language-activated cerebral blood oxygenation and hemodynamic changes of the left prefrontal cortex in poststroke aphasic patients: a near-infrared spectroscopy study," Stroke 29(7), 1299-304 (1998).

46. J. S. Wyatt et al., "Quantification of cerebral oxygenation and haemodynamics in sick newborn infants by near infrared spectrophotometry," Lancet 328(8515), 1063-1066 (1986).

47. D. Canova et al., "Inconsistent detection of changes in cerebral blood volume by near infrared spectroscopy in standard clinical tests," J. Appl. Physiol. 110(6), 1646-1655 (2011).

48. G. E. Strangman, Q. Zhang, and Z. Li, "Scalp and skull influence on near infrared photon propagation in the Colin27 brain template," NeuroImage 85(1), 136-149 (2014).

49. S. Brigadoi and R. J. Cooper, "How short is short? Optimum sourcedetector distance for short-separation channels in functional near-infrared spectroscopy," Neurophotonics 2(2), 025005 (2015).

50. L. A. Henderson, "Brain responses associated with the Valsalva maneuver revealed by functional magnetic resonance imaging," $J$. Neurophysiol. 88, 3477-3486 (2002).

Biographies for the authors are not available. 